\title{
Intake of dietary antioxidants is inversely associated with biomarkers of oxidative stress among men with prostate cancer
}

\author{
Terrence M. Vance $^{1}$, Gissou Azabdaftari ${ }^{2}$, Elena A. Pop ${ }^{2}$, Sang Gil Lee ${ }^{1}$, L. Joseph Su ${ }^{3}$, \\ Elizabeth T. H. Fontham ${ }^{4}$, Jeannette T. Bensen ${ }^{5}$, Susan E. Steck ${ }^{6}$, Lenore Arab $^{7}$, \\ James L. Mohler ${ }^{2,5}$, Ming-Hui $\mathrm{Chen}^{8}$, Sung I. Koo ${ }^{1}$ and Ock K. Chun ${ }^{1 *}$ \\ ${ }^{1}$ Department of Nutritional Sciences, University of Connecticut, Storrs, CT 06269-4017, USA \\ ${ }^{2}$ Roswell Park Cancer Institute, Buffalo, NY 14263, USA \\ ${ }^{3}$ Epidemiology and Genomics Research Program, Division of Cancer Control and Population Sciences, National Cancer \\ Institute, Bethesda, MD 20892, USA \\ ${ }^{4}$ School of Public Health, Louisiana State University Health Sciences Center, New Orleans, LA 70112, USA \\ ${ }^{5}$ School of Public Health, University of North Carolina at Chapel Hill, Chapel Hill, NC 27599, USA \\ ${ }^{6}$ Department of Epidemiology and Biostatistics, Cancer Prevention and Control Program, University of South Carolina, \\ Columbia, SC 29208, USA \\ ${ }^{7}$ David Geffen School of Medicine, University of California, Los Angeles, CA 90095, USA \\ ${ }^{8}$ Department of Statistics, University of Connecticut, Storrs, CT 06269, USA
}

(Submitted 23 July 2015 - Final revision received 23 September 2015 - Accepted 29 September 2015 - First published online 2 November 2015)

\section{Abstract}

Prostate cancer is the most common non-cutaneous cancer and the second leading cause of cancer-related mortality among men in the USA. Growing evidence suggests that oxidative stress is involved in the development and progression of prostate cancer. In this study, the association between antioxidants from diet and supplements and biomarkers of oxidative stress in blood ( $n$ 278), urine ( $n$ 298) and prostate tissue $(n 55)$ were determined among men from the North Carolina-Louisiana Prostate Cancer Project. The association between antioxidant intake and oxidative stress biomarkers in blood and urine was determined using linear regression, adjusting for age, race, prostate cancer aggressiveness and smoking status. Greater antioxidant intake was found to be associated with lower urinary 8-isoprostane concentrations, with a $10 \%$ increase in antioxidant intake corresponding to an unadjusted $1.1 \%$ decrease in urinary 8 -isoprostane levels $(95 \% \mathrm{CI}-1.7,-0.3 \%$; $P$ value $<0.01)$ and an adjusted $0.6 \%$ decrease $(95 \% \mathrm{CI}-1.4,0.2 \% ; P$ value $=0.16)$. In benign prostate tissue, thioredoxin 1 was inversely associated with antioxidant intake $(P=0.02)$. No significant associations were found for other blood or urinary biomarkers or for malignant prostate tissue. These results indicate that antioxidant intake may be associated with less oxidative stress among men diagnosed with prostate cancer.

Key words: Prostate cancer: Diets: Antioxidants: Oxidative stress

Prostate cancer is the most common non-cutaneous cancer and the second leading cause of cancer-related mortality in men in the USA, with an estimated 220800 new cases and 27540 deaths in $2015^{(1)}$. The specific causes of prostate cancer have not yet been determined, but several risk factors have been identified for the disease - namely, family history, age and race ${ }^{(2,3)}$. However, the mechanisms by which these and other risk factors, such as lifestyle, contribute to prostate cancer are not clear, and there is likely a great degree of heterogeneity in the causes of the disease.

At the cellular level, a growing body of evidence indicates that oxidative stress is involved in prostate carcinogenesis ${ }^{(4)}$.
Reactive oxygen species (ROS) can promote carcinogenesis by causing oxidative damage to DNA and macromolecules within cells, altering signal transduction pathways, and promoting a malignant phenotype ${ }^{(4)}$. Furthermore, antioxidant enzyme genotype has been associated with cancer risk ${ }^{(5)}$, and prostate cancer risk may be modified by interactions between antioxidant enzyme genotype and dietary antioxidants ${ }^{(6,7)}$. Men diagnosed with prostate cancer have been shown to have greater oxidative stress, lower antioxidant enzyme activity ${ }^{(8)}$ and greater levels of urinary isoprostanes - a biomarker of oxidative stress and lipid peroxidation ${ }^{(9,10)}$. Furthermore, in vitro experiments have implicated the production of ROS in

Abbreviations: PCaP, North Carolina-Louisiana Prostate Cancer Project; Trx1, thioredoxin 1.

* Corresponding author: Associate Professor O. K. Chun, email ock.chun@uconn.edu 
the aggressiveness of prostate cancer cells ${ }^{(11)}$. Thus, relatively consistent evidence suggests an association between oxidative stress and prostate cancer.

Oxidative stress is regulated in both benign and malignant cells via several non-enzymatic and enzymatic antioxidant mechanisms ${ }^{(4)}$. Malignant cells have greater levels of ROS and compensatory increases in antioxidant enzymes in order to tolerate increased oxidative stress ${ }^{(12)}$. However, the evidence for a relationship between dietary antioxidants and prostate cancer is inconsistent in humans ${ }^{(13)}$, which is partly due to the relatively small number of dietary antioxidants studied and their diversity in structure and possible mechanisms of action. Dietary antioxidants such as flavonoids have been shown to reduce oxidative damage ${ }^{(14)}$ and promote DNA repair ${ }^{(15)}$ in prostate cancer cells in vitro. Thus, dietary antioxidants could have some effect on prostate cancer, possibly by affecting oxidative stress.

The discrepant findings regarding prostate cancer and dietary antioxidants prompted this study of dietary antioxidant intake using a cumulative index of the antioxidant capacity of diet and supplements ${ }^{(16)}$. A cumulative measure of antioxidant intake from diet and supplements may circumvent issues of multiplicity in associations between individual dietary antioxidants and disease risk. Such an estimate of dietary antioxidant intake has been studied recently with regard to prostate cancer incidence ${ }^{(17)}$; however, little is known about the relationship between dietary antioxidants and levels of oxidative stress among prostate cancer patients. Therefore, the objectives of this study were to measure the dietary antioxidant intake and biomarkers of oxidative stress of men diagnosed with incident prostate cancer and to determine whether antioxidant intake is inversely related to oxidative stress.

\section{Methods}

The present study used data collected by the North CarolinaLouisiana Prostate Cancer Project (PCaP), a population-based study of incident prostate cancer ${ }^{(18)}$. In brief, men between 40 and 79 years of age with a first diagnosis of histologically confirmed adenocarcinoma of the prostate on or after 1 July 2004, were eligible to participate in the PCaP. Men had to be able to complete the study interview in English and could not live in an institution or nursing home, be cognitively impaired, under the influence of alcohol, severely medicated or apparently psychotic. Men must have self-identified as either African-American or black or Caucasian or white when responding to the question 'What is your race?' This project was approved by the institutional review boards at the Department of Defense Prostate Cancer Research Program, the University of North Carolina and Louisiana State University. The institutional review board at the University of Connecticut also approved this study.

\section{Dietary antioxidants}

Dietary data were collected using a modified version of the National Cancer Institute Diet History Questionnaire (DHQ) ${ }^{(19)}$, which assessed average daily dietary intake for 1 year before diagnosis, and supplement data were collected via a questionnaire administered by a PCaP nurse. Data regarding flavonoid and proanthocyanidin contents of foods were added to the DHQ database using Nutrition Data System for Research, version 2011 (Nutrition Coordinating Center, University of Minnesota). The 2,2'-azino-bis(3-ethylbenzothiazoline-6-sulphonic acid) (ABTS) radical anion scavenging activity assay was used previously to measure the vitamin $\mathrm{C}$ equivalent (VCE) antioxidant capacities of individual antioxidants ${ }^{(20)}$ and the values were applied to the $\mathrm{PCaP}$ diet and supplement data. Antioxidant intake was calculated from the VCE antioxidant capacity of forty-two dietary antioxidants (carotenoids, vitamins $\mathrm{C}$ and $\mathrm{E}$, flavonoids, proanthocyanidins and isoflavones) and five antioxidants from dietary supplements (vitamin C, $\alpha$-tocopherol, $\beta$-carotene, lycopene, and lutein and zeaxanthin).

\section{Blood and urinary biomarkers of oxidative stress}

Urine, erythrocyte, plasma and tissue samples were available from 298, 271, 279 and fifty-nine men, respectively, and these samples were collected from men who reported not having undergone any treatment for their prostate cancer, as it was thought that treatment might influence reporting of dietary recall or oxidative stress. Blood and urine samples were collected from PCaP research subjects at the time of interview, centrifuged, aliquoted and stored at $-80^{\circ} \mathrm{C}$ until analysis. The activities of erythrocyte glutathione peroxidase, glutathione $S$-transferase, glutathione reductase and superoxide dismutase, as well as the concentrations of plasma malondialdehyde and urinary 8-isoprostane and creatinine, were measured using commercial kits (Cayman Chemical Company) according to the manufacturer's instructions. Plasma antioxidant capacity was measured using the ABTS radical anion scavenging activity assay. In brief, 2,2'-azobis(2-amidinopropane) $\mathrm{HCl}$ and ABTS were dissolved in potassium phosphate buffer solution and heated to $70^{\circ} \mathrm{C}$ for $30 \mathrm{~min}$. The mixture was then cooled to room temperature and diluted to reach an absorbance of $0.650 \pm 0.020$ at $734 \mathrm{~nm}$. This solution was added to the plasma samples, and the absorbance was measured at $734 \mathrm{~nm}$ after 10 min. Plasma antioxidant capacity was calculated in VCE using a vitamin $\mathrm{C}$ standard curve.

\section{Prostate tissue thioredoxin 1}

Paraffin-embedded prostate tissue biopsy specimens were selected from PCaP research subjects who were untreated at the time of sample collection to provide an approximately equal proportion of subjects by site, race and aggressiveness level to yield a subsample of fifty-nine tissue sections ( 5 - $\mu$ m thickness) for analysis. Sections were deparaffinised, rehydrated under an alcohol gradient and antigen retrieved using Reveal Decloaker (Biocare Medical) for $15 \mathrm{~min}$ at $110^{\circ} \mathrm{C}$ in a Decloaking Chamber (Biocare Medical). Sections were blocked for endogenous peroxidase activity using $3 \% \mathrm{H}_{2} \mathrm{O}_{2}$, rinsed with deionised, distilled water for $10 \mathrm{~min}$ at room temperature, blocked with normal goat serum for $1 \mathrm{~h}$ and incubated overnight at $4^{\circ} \mathrm{C}$ with rabbit anti-thioredoxin 1 (1:1000; Cell Signaling). The next day, 
sections were incubated for $30 \mathrm{~min}$ at room temperature with Rabbit SignalStain Boost IHC Reagent, the enzymatic activity was revealed using diaminobenzidine (Sigma-Aldrich), counterstained with haematoxylin (Vector Laboratories) and mounted using a permanent mounting medium. Images of the stained sections were collected using a Leica DFC0425C camera (Leica) mounted on a Leica DMRA2 microscope (Leica) equipped with an automated stage. Prostate cancer Gleason's scores of all the biopsy sections were determined by a genitourinary pathologist (G. A.). Levels of thioredoxin 1 (Trx1) in benign and malignant tissue were visually scored by GA in a blinded manner. Slides were scored from 0 to 3 , with 0 indicating absence of staining and 1 through 3 indicating greater intensity of staining.

A total of fifty-nine prostate biopsy specimens were randomly selected for analyses. Some of the samples were excluded from the analyses because sections that contained malignant tissue were unavailable for four biopsies and sections containing benign tissue were unavailable for three biopsies. The final number of prostate biopsy specimens used for the analyses of Trx1 were fifty-five malignant tissue and fifty-four benign tissue samples.

\section{Statistical analyses}

All the statistical analyses were performed using SAS software, version 9.3 (SAS Institute Inc.). Men were ranked into three groups based on dietary antioxidant intake, and descriptive statistics are reported for each group (Tables 1 and 2). Antioxidant intake was adjusted for average energy intake using the residual method ${ }^{(21)}$. Linear regression was used to determine the association between antioxidant intake from diet and supplements and biomarkers of oxidative stress, adjusting for average energy intake, age, race, prostate cancer aggressiveness and smoking status. On the basis of residual plots, antioxidant intake, plasma glutathione $S$-transferase activity, erythrocyte glutathione reductase activity and urinary 8 -isoprostane levels were log transformed to improve linearity. As for all regression models antioxidant intake was log transformed, the associations between intake of antioxidants and biomarkers are reported as the predicted change in the biomarker for a $10 \%$ increase in dietary antioxidants. As the range of antioxidant intake might differ between supplement users and non-users and as supplement use might be associated with other confounders, we tested for an interaction between antioxidant intake and an indicator variable that dichotomised supplement users and non-users. This interaction allows for testing whether the association between antioxidant intake and biomarkers differs between supplement users and non-users. The association between Trx1 and categories of antioxidant intake was determined using Fisher's exact test. For all the analyses, a $P$ value of $<0.05$ was used to determine statistical significance.

\section{Results}

The range in antioxidant intake ( $\mathrm{mg} \mathrm{VCE} / \mathrm{d}$ ) among men in tertiles was 61-388 for tertile 1,389-804 for tertile 2 and
829-4895 for tertile 3. Descriptive statistics for research subjects are presented in Table 1. A greater proportion of white, older and overweight and obese men, as well as men who reported having a prostatic-specific antigen test, reported greater antioxidant intake. The median levels of biomarkers by dietary antioxidant intake are reported in Table 2. Plasma malondialdehyde was the lowest and erythrocyte glutathione peroxidase activity was the highest among men in the highest tertile of antioxidant intake. Urinary 8-isoprostane level was inversely associated with dietary antioxidant intake, with levels of 8-isoprostane decreasing across tertiles.

In linear regression models of antioxidant intake predicting biomarkers of oxidative stress, there was no statistically significant interaction between supplement use and antioxidant intake in both unadjusted and adjusted analyses, which indicates that the association between antioxidant intake and biomarkers did not significantly differ between users and non-users of dietary supplements. There was little evidence of an association between dietary antioxidant intake and biomarkers from plasma or erythrocytes (Table 3). In unadjusted analyses, urinary 8-isoprostane was significantly associated with antioxidant intake, with a $10 \%$ increase in antioxidant intake corresponding to a $1.1 \%$ decrease in urinary 8 -isoprostane (95\% CI $-1.9,-0.3 \% ; P$ value $<0.01)$. After adjusting for age, race, aggressiveness and smoking status, this association was no longer statistically significant; however, the direction of the association was similar, with a $10 \%$ increase in antioxidant intake associated with a $0.6 \%$ decrease in urinary 8 -isoprostane (95\% CI $-1.4,0.2 \% ; \quad P$ value $=0 \cdot 16)$. After adjustment, there was some evidence of a weak inverse association between antioxidant intake and erythrocyte glutathione $S$-transferase activity, with a $10 \%$ increase in antioxidant intake corresponding to a predicted decrease in erythrocyte glutathione $S$-transferase activity of 0.5 (95\% CI $-1.0,0.0 \mathrm{nmol} / \mathrm{min}$ per $\mathrm{ml} ; P$ value $=0.05)$. There was no evidence of an association between Trx1 in malignant prostate tissue and dietary antioxidant intake $(P$ value $=0.90)$ (Table 4); however, there was evidence of an inverse association between antioxidant intake and Trx1 in benign prostate tissue $(P$ value $=0 \cdot 02)$.

\section{Discussion}

Men with prostate cancer have been found to have low blood levels of dietary antioxidants ${ }^{(23)}$, decreased activity of endogenous antioxidant enzymes and increased levels of lipid peroxidation $^{(8,24)}$. These findings could indicate either greater oxidative stress, resulting in depletion of antioxidants, or lower levels of antioxidants, resulting in increased oxidative stress and lipid peroxidation. Furthermore, levels of antioxidants in the blood may interact with genetic variants of cyclo-oxygenase- 2 and $I L-8$, which are involved in inflammation $^{(25)}$, and human oxoguanine glycosylase 1 and $X$-ray repair cross-complementing group 1 , which are involved in repairing DNA damage ${ }^{(7,26)}$. Thus, it is plausible that dietary antioxidants may influence prostate cancer development and progression with oxidative stress-mediated damage. 
Table 1. Socio-demographic and clinical characteristics by tertile (T) of antioxidant intake (Numbers and percentages)

\begin{tabular}{|c|c|c|c|c|c|c|}
\hline & \multicolumn{6}{|c|}{ Antioxidant intake } \\
\hline & \multicolumn{2}{|c|}{$\mathrm{T} 1$} & \multicolumn{2}{|c|}{$\mathrm{T} 2$} & \multicolumn{2}{|c|}{ T3 } \\
\hline & $n$ & $\%$ & $n$ & $\%$ & $n$ & $\%$ \\
\hline \multicolumn{7}{|l|}{ Age (years) } \\
\hline$<50$ & 6 & $46 \cdot 2$ & 4 & $30 \cdot 8$ & 3 & $23 \cdot 1$ \\
\hline $51-60$ & 24 & $34 \cdot 3$ & 22 & 31.4 & 24 & $34 \cdot 3$ \\
\hline $61-70$ & 25 & 29.4 & 27 & 31.8 & 33 & 38.8 \\
\hline$>70$ & 11 & 20 & 24 & 43.6 & 20 & $36 \cdot 4$ \\
\hline \multicolumn{7}{|l|}{ Race } \\
\hline White & 32 & 26.9 & 38 & 31.9 & 49 & $41 \cdot 2$ \\
\hline Black & 34 & $32 \cdot 7$ & 39 & $37 \cdot 5$ & 31 & $29 \cdot 8$ \\
\hline \multicolumn{7}{|l|}{$\mathrm{BMI}$} \\
\hline Normal & 16 & 36.4 & 16 & 36.4 & 12 & $27 \cdot 3$ \\
\hline Overweight & 26 & 28 & 34 & $36 \cdot 6$ & 33 & 35.5 \\
\hline Obese & 24 & $27 \cdot 9$ & 27 & 31.4 & 35 & $40 \cdot 7$ \\
\hline \multicolumn{7}{|l|}{ Smoking history } \\
\hline Non-smoker & 16 & $21 \cdot 6$ & 27 & $36 \cdot 5$ & 31 & 41.9 \\
\hline Former smoker & 35 & $31 \cdot 8$ & 35 & 31.8 & 40 & $36 \cdot 4$ \\
\hline Smoker & 15 & 38.5 & 15 & 38.5 & 9 & $23 \cdot 1$ \\
\hline \multicolumn{7}{|l|}{ Aggressiveness* } \\
\hline Low & 43 & $28 \cdot 7$ & 51 & 34 & 56 & $37 \cdot 3$ \\
\hline Intermediate & 16 & 31.4 & 19 & $37 \cdot 3$ & 16 & 31.4 \\
\hline High & 7 & 31.8 & 7 & 31.8 & 8 & $36 \cdot 4$ \\
\hline \multicolumn{7}{|l|}{ PSA test } \\
\hline Yes & 44 & $25 \cdot 6$ & 57 & $33 \cdot 1$ & 71 & $41 \cdot 3$ \\
\hline No & 22 & $43 \cdot 1$ & 20 & $39 \cdot 2$ & 9 & $17 \cdot 6$ \\
\hline \multicolumn{7}{|l|}{ Family history† } \\
\hline Yes & 10 & $41 \cdot 7$ & 4 & $16 \cdot 7$ & 10 & $41 \cdot 7$ \\
\hline No & 56 & $28 \cdot 1$ & 73 & $36 \cdot 7$ & 70 & $35 \cdot 2$ \\
\hline
\end{tabular}

PSA, prostatic-specific antigen.

* High aggressive defined as Gleason's sum $\geq 8$ or PSA $>20 \mathrm{ng} / \mathrm{ml}$ or Gleason's sum $=7$ and clinical stage T3-T4; low aggressive as Gleason's sum $<7$ and clinical stage T1-T2 and PSA $<10 \mathrm{ng} / \mathrm{ml}$; all other cases were defined as intermediate aggressive.

$\dagger$ Defined as having at least one relative diagnosed with prostate cancer before 60 years of age.

Table 2. Antioxidant biomarkers by tertile $(\mathrm{T})$ of antioxidant intake (Medians and interquartile ranges (IQR))

\begin{tabular}{|c|c|c|c|c|c|c|}
\hline & \multicolumn{6}{|c|}{ Antioxidant intake } \\
\hline & \multicolumn{2}{|c|}{$\mathrm{T} 1$} & \multicolumn{2}{|c|}{ T2 } & \multicolumn{2}{|c|}{ T3 } \\
\hline & Median & IQR & Median & IQR & Median & IQR \\
\hline \multicolumn{7}{|l|}{ Plasma } \\
\hline TAC (mg VCE/l) & 63.9 & $57 \cdot 9,70 \cdot 0$ & 63.8 & $53 \cdot 0,69 \cdot 9$ & $63 \cdot 1$ & $53 \cdot 5,68 \cdot 8$ \\
\hline MDA ( $\mu \mathrm{M})$ & $207 \cdot 7$ & $170 \cdot 9,243 \cdot 5$ & $209 \cdot 2$ & $177 \cdot 5,238 \cdot 7$ & $197 \cdot 7$ & $170 \cdot 2,255 \cdot 6$ \\
\hline GST activity (nmol/min per ml) & 14.4 & $13 \cdot 0,16 \cdot 3$ & $15 \cdot 3$ & $12 \cdot 4,17 \cdot 5$ & $15 \cdot 9$ & $13 \cdot 9,18 \cdot 3$ \\
\hline \multicolumn{7}{|l|}{ Erythrocytes } \\
\hline GST activity (nmol/min per $\mathrm{ml})$ & $46 \cdot 1$ & $33.4,61.6$ & 44.9 & $27 \cdot 2,66 \cdot 6$ & 43.3 & $25 \cdot 8,63 \cdot 2$ \\
\hline GPx activity (nmol/min per $\mathrm{ml})$ & $764 \cdot 1$ & $438 \cdot 1,985 \cdot 7$ & $745 \cdot 0$ & $483 \cdot 9,995 \cdot 8$ & $841 \cdot 8$ & $556 \cdot 8,1031.5$ \\
\hline $\mathrm{GR}$ activity $(\mathrm{nmol} / \mathrm{min}$ per $\mathrm{ml})$ & 207.8 & $151 \cdot 8,290 \cdot 3$ & $223 \cdot 1$ & $170 \cdot 6,285 \cdot 3$ & $224 \cdot 1$ & $157 \cdot 9,309 \cdot 2$ \\
\hline SOD activity $(\mathrm{U} / \mathrm{ml})^{*}$ & $76 \cdot 3$ & $69 \cdot 8,78 \cdot 8$ & $73 \cdot 2$ & $68.9,78.8$ & 74.7 & $69.4,78 \cdot 8$ \\
\hline \multicolumn{7}{|l|}{ Urine } \\
\hline 8-Isoprostane ( $\mathrm{ng} / \mathrm{mmol}$ creatinine) & $140 \cdot 0$ & $103 \cdot 1,195 \cdot 5$ & $121 \cdot 2$ & $85 \cdot 2,193.5$ & $116 \cdot 2$ & $82 \cdot 5,151 \cdot 8$ \\
\hline
\end{tabular}

TAC, total antioxidant capacity; VCE, vitamin C equivalents; MDA, malondialdehyde; GST, glutathione S-transferase; GPx, glutathione peroxidase; GR, glutathione reductase; SOD, superoxide dismutase.

* One unit of SOD is defined as the amount of enzyme needed to exhibit $50 \%$ dismutation of the superoxide radical.

The present study was unable to establish the direction of cause and effect, due to its cross-sectional design. However, the significant, inverse associations between dietary antioxidants and levels of urinary 8 -isoprostane suggest that the oxidative stress of men with prostate cancer may in part depend on diet. This may indicate that men with greater intake of dietary antioxidants have greater inhibition of free radicals and subsequently less lipid peroxidation ${ }^{(27)}$. It is also possible that the inverse association observed may reflect some non-antioxidant mechanisms of dietary components assumed to function as 
Table 3. Association between oxidative stress biomarkers and dietary antioxidants from linear regression (Changes and $95 \%$ confidence intervals)

\begin{tabular}{|c|c|c|c|c|c|c|}
\hline \multirow[b]{2}{*}{ Biomarkers } & \multicolumn{3}{|c|}{ Unadjusted } & \multicolumn{3}{|c|}{ Adjusted* $^{*}$} \\
\hline & Change† & $95 \% \mathrm{Cl}$ & $P$ & Change & $95 \% \mathrm{Cl}$ & $P$ \\
\hline \multicolumn{7}{|l|}{ Plasma } \\
\hline TAC (mg VCE/l) & 0.0 & $-0.2,0.2$ & 0.81 & $-0 \cdot 1$ & $-0.3,0.1$ & 0.32 \\
\hline MDA ( $\mu \mathrm{M})$ & 0.3 & $-0 \cdot 6,1 \cdot 3$ & 0.49 & 0.4 & $-0.6,1.5$ & 0.43 \\
\hline GST activity (nmol/min per ml) & $0.3 \%$ & $-0.1,0.8 \%$ & 0.15 & $0.3 \%$ & $-0.2,0.8 \%$ & $0 \cdot 18$ \\
\hline \multicolumn{7}{|l|}{ Erythrocytes } \\
\hline GST activity (nmol/min per ml) & $-0 \cdot 3$ & $-0.8,0.2$ & 0.18 & -0.5 & $-1 \cdot 0,0 \cdot 0$ & 0.05 \\
\hline GPx activity (nmol/min per ml) & 4.9 & $-0.8,10 \cdot 6$ & 0.09 & $3 \cdot 7$ & $-2 \cdot 4,9 \cdot 9$ & 0.24 \\
\hline GR activity (nmol/min per ml) & $0.0 \%$ & $-0.8,0.9 \%$ & 0.94 & $-0.3 \%$ & $-1 \cdot 2,0.6 \%$ & 0.55 \\
\hline SOD activity $(\mathrm{U} / \mathrm{ml})$ & 0.0 & $-0.1,0.2$ & 0.63 & 0.0 & $-0.2,0.2$ & 0.73 \\
\hline \multicolumn{7}{|l|}{ Urine } \\
\hline 8-Isoprostane (ng/mmol creatinine) & $-1.1 \%$ & $-1.9,-0.3 \%$ & $<0.01$ & $-0.6 \%$ & $-1.4,0.2 \%$ & 0.16 \\
\hline
\end{tabular}

TAC, total antioxidant capacity; VCE, vitamin C equivalents; MDA, malondialdehyde; GST, glutathione S-transferase; GPx, glutathione peroxidase; GR, glutathione reductase; SOD, superoxide dismutase.

* Adjusted for age, race, aggressiveness and smoking status.

† Predicted change in biomarker for a $10 \%$ increase in dietary antioxidants.

Table 4. Thioredoxin 1 (Trx1) levels in malignant and benign tissue by tertile $(T)$ of antioxidant intake* (Numbers and percentages)

\begin{tabular}{|c|c|c|c|c|c|c|c|}
\hline & \multicolumn{6}{|c|}{ Antioxidant intake } & \multirow[b]{3}{*}{$P \dagger$} \\
\hline & \multicolumn{2}{|c|}{ T1 } & \multicolumn{2}{|c|}{ T2 } & \multicolumn{2}{|c|}{ T3 } & \\
\hline & $n$ & $\%$ & $n$ & $\%$ & $n$ & $\%$ & \\
\hline \multicolumn{8}{|c|}{ Malignant Trx1 } \\
\hline 0 & 0 & 0.0 & 2 & $33 \cdot 3$ & 2 & $66 \cdot 7$ & 0.90 \\
\hline 1 & 3 & 28.6 & 3 & $42 \cdot 9$ & 2 & 28.6 & \\
\hline 2 & 8 & 35.0 & 8 & $25 \cdot 0$ & 8 & $40 \cdot 0$ & \\
\hline 3 & 5 & 33.3 & 7 & $26 \cdot 7$ & 7 & $40 \cdot 0$ & \\
\hline \multicolumn{8}{|c|}{ Benign Trx1 } \\
\hline 0 & 0 & 0.0 & 2 & $100 \cdot 0$ & 0 & 0.0 & 0.02 \\
\hline 1 & 10 & 30.8 & 13 & 34.6 & 9 & 34.6 & \\
\hline 2 & 3 & $20 \cdot 0$ & 4 & $20 \cdot 0$ & 10 & $60 \cdot 0$ & \\
\hline 3 & 3 & $100 \cdot 0$ & 0 & 0.0 & 0 & 0.0 & \\
\hline
\end{tabular}

* Trx1 data from this sample has been published in Vance et al. ${ }^{(22)}$.

$\dagger P$ value from Fisher's exact test.

antioxidants in vivo. There was no observed difference in this association among men with different levels of prostate cancer aggressiveness (data not shown). This, combined with the limitations inherent to a cross-sectional study, does not allow for the determination of whether the association between diet and oxidative stress observed among these men will reflect meaningful clinical outcome measures, such as disease progression and mortality.

There was little or no evidence of an association between antioxidant intake and the activity of plasma or erythrocyte antioxidant enzymes, and the association between dietary antioxidants and erythrocyte glutathione $S$-transferase could be due to chance. There are several explanations for this absence of effect. Arsova-Sarafinovska et al. ${ }^{(8)}$ found that erythrocyte antioxidant enzyme activity was decreased in prostate cancer patients compared with controls. As the present study included only men diagnosed with prostate cancer, differences between healthy men and those with prostate cancer may be greater than differences among men with prostate cancer. Other studies have demonstrated that polymorphisms in the genes of antioxidant enzymes are related to prostate cancer ${ }^{(5,6)}$; antioxidant enzyme activity, as assessed in this study, may not be sensitive enough to capture functional differences in antioxidant enzymes that result from differences in amino acid sequences. Furthermore, antioxidants in plasma or erythrocytes may not reflect prostate tissue antioxidant activity. The null finding regarding diet and malondialdehyde could reflect the method of measurement. Malondialdehyde was measured using the thiobarbituric acid reactive substances method, which may not be a sensitive measure of lipid peroxidation and oxidative stress ${ }^{(28)}$.

The primary strength of this study was the use of a prostatic biomarker to assess prostate tissue redox status. Trx1 in benign prostate tissue was found to be inversely associated with antioxidant intake (Table 4). Trx1 is thought to be involved in prostate carcinogenesis and may indicate redox imbalance in prostate cancer ${ }^{(29)}$. Thioredoxin reductase 1 (TrxR1) transfers electrons from NADPH to Trx1, a process that occurs in most living cells and is essential for the maintenance of cellular redox 
status ${ }^{(30)}$. Both Trx1 and TrxR1 are increased in prostate cancer cells ${ }^{(29,31)}$, with a greater proportion of Trx1 in an oxidised state, which may reflect redox imbalance and response to greater levels of oxidative stress. Expression of TrxR1, the enzyme responsible for maintaining Trx1 in a reduced form, has been shown to be increased in castration-resistant prostate cancer ${ }^{(31)}$. Reduced Trx1 binds to apoptosis signal-regulating kinase 1 and regulates cell death ${ }^{(32)}$; thus, redox imbalance may promote cancer cell survival. Taken together, greater levels of Trx1 correspond to more aggressive forms of prostate cancer. The inverse association between antioxidant intake and Trx1 requires further research to determine whether this association might affect disease severity or progression, particularly given the relatively small number of tissue samples analysed in this study. Relatively few studies have been conducted on the thioredoxin system and prostate cancer; moreover, although this system appears to play an important role in prostate cancer, the extent to which this system affects prostate cancer development and progression in humans remains uncertain.

The present study has several limitations. The cross-sectional design of the study prohibits establishing a cause-effect relationship between antioxidant intake and urinary 8-isoprostane and prostate tissue Trx1. Dietary data were collected after diagnosis and knowledge of disease may have biased responses by research subjects, although this would likely have been non-differential. The number of research subjects included in the study was relatively low, particularly with regard prostate tissue Trx1, which should be taken into consideration when interpreting these results. The apparent inverse association between antioxidant intake and $\operatorname{Trx} 1$ in benign prostate tissue is tenuous based on available evidence. One interpretation of the current data is that greater antioxidant intake reduces oxidative stress, thereby inhibiting the activation of nuclear factor (erythroid-derived 2)-like 2 and its promotion of Trx1 transcription $^{(33)}$. However, we did not measure the proportion of oxidised and reduced Trx1 in prostate tissue, and this additional data would have shed light on the observed associations with Trx1 in the present study and oxidative stress ${ }^{(29)}$. Additional work is needed to confirm this association and determine whether intake of antioxidants can influence the oxidation state of $\operatorname{Trx} 1$ and oxidative stress in the prostate, and whether these effects influence relevant clinical outcomes such as prostate cancer mortality.

This study demonstrated that intake of antioxidants was associated with less oxidative stress among men with incident prostate cancer. The results of this study and others warrant additional research in humans on the mechanisms underlying the relationship between dietary antioxidants and prostate tissue redox status and carcinogenesis, as well as determining whether this relationship may influence disease severity, progression and recurrence.

\section{Acknowledgements}

The authors thank the staff, advisory committees and research subjects participating in the North Carolina-Louisiana Prostate
Cancer Project study for their important contributions. The authors thank John Stocking and Sarah McEvoy for technical assistance with immunostaining. The authors acknowledge the Roswell Park Cancer Institute Department of Urology Tissue Microarray and Immuno-Analysis Core for the tissue processing, storage and sample disbursement and acknowledge the University of North Carolina Biospecimen Facility and the Louisiana State University Health Sciences Center Pathology Laboratory for the blood sample processing, storage and sample disbursement (https://genome.unc.edu/bsp).

This study was supported by the National Institutes of Health Cancer Epidemiology small grant no. 1R03CA159421-01A1 and Department of Defense contract DAMD 17-03-2-0052.

T. M. V. performed the experimental work and data analyses and prepared the manuscript. S. G. L. performed the experimental work. G. A. and E. A. P. measured thioredoxin 1 and contributed to the manuscript. L. J. S., E. T. H. F., J. T. B., J. L. M., S. E. S. and L. A. helped supervise the field activities, designed the study's analytic strategy and contributed to the manuscript. O. K. C. designed the study and directed its implementation, including quality assurance and control. S. I. K. reviewed the manuscript. M.-H. C. provided statistical consultation on data analyses and interpretation of the results.

The authors have no conflicts of interest regarding this manuscript.

\section{References}

1. Siegel RL, Miller KD \& Jemal A (2015) Cancer statistics, 2015. CA Cancer J Clin 65, 5-29.

2. Brawley OW (2012) Prostate cancer epidemiology in the United States. World J Urol 30, 195-200.

3. Patel AR \& Klein EA (2009) Risk factors for prostate cancer. Nat Clin Pract Urol 6, 87-95.

4. Paschos A, Pandya R, Duivenvoorden WC, et al. (2013) Oxidative stress in prostate cancer: changing research concepts towards a novel paradigm for prevention and therapeutics. Prostate Cancer Prostatic Dis 16, 217-225.

5. Klaunig JE, Kamendulis LM \& Hocevar BA (2010) Oxidative stress and oxidative damage in carcinogenesis. Toxicol Pathol 38, 96-109.

6. Li H, Kantoff PW, Giovannucci E, et al. (2005) Manganese superoxide dismutase polymorphism, prediagnostic antioxidant status, and risk of clinical significant prostate cancer. Cancer Res 65, 2498-2504.

7. Goodman M, Bostick RM, Ward KC, et al. (2006) Lycopene intake and prostate cancer risk: effect modification by plasma antioxidants and the XRCC1 genotype. Nutr Cancer 55, 13-20

8. Arsova-Sarafinovska Z, Eken A, Matevska N, et al. (2009) Increased oxidative/nitrosative stress and decreased antioxidant enzyme activities in prostate cancer. Clin Biochem $\mathbf{4 2}$, 1228-1235.

9. Brys M, Morel A, Forma E, et al. (2013) Relationship of urinary isoprostanes to prostate cancer occurrence. Mol Cell Biochem 372, 149-153.

10. Barocas DA, Motley S, Cookson MS, et al. (2011) Oxidative stress measured by urine F2-isoprostane level is associated with prostate cancer. J Urol 185, 2102-2107.

11. Kumar B, Koul S, Khandrika L, et al. (2008) Oxidative stress is inherent in prostate cancer cells and is required for aggressive phenotype. Cancer Res 68, 1777-1785. 
12. Cairns RA, Harris IS \& Mak TW (2011) Regulation of cancer cell metabolism. Nat Rev Cancer 11, 85-95.

13. Vance TM, Su J, Fontham ET, et al. (2013) Dietary antioxidants and prostate cancer: a review. Nutr Cancer 65, 793-801.

14. Sharma H, Kanwal R, Bhaskaran N, et al. (2014) Plant flavone apigenin binds to nucleic acid bases and reduces oxidative DNA damage in prostate epithelial cells. PLOS ONE 9, e91588.

15. Gao K, Henning SM, Niu Y, et al. (2006) The citrus flavonoid naringenin stimulates DNA repair in prostate cancer cells. J Nutr Biochem 17, 89-95.

16. Serafini M \& Del Rio D (2004) Understanding the association between dietary antioxidants, redox status and disease: is the Total Antioxidant Capacity the right tool? Redox Rep 9 , 145-152.

17. Russnes KM, Wilson KM, Epstein MM, et al. (2013) Total antioxidant intake in relation to prostate cancer incidence in the health professionals follow up study. Int J Cancer 134, $1156-1165$.

18. Schroeder JC, Bensen JT, Su LJ, et al. (2006) The North Carolina-Louisiana Prostate Cancer Project (PCaP): methods and design of a multidisciplinary population-based cohort study of racial differences in prostate cancer outcomes. Prostate 66, 1162-1176.

19. National Institutes of Health Applied Research Program, National Cancer Institute (2007) Diet History Questionnaire, Version 1.0, National Institutes of Health Applied Research Program. Rockville, MD: National Cancer Institute.

20. Floegel A, Kim DO, Chung SJ, et al. (2010) Development and validation of an algorithm to establish a total antioxidant capacity database of the US diet. Int J Food Sci Nutr 61, 600-623.

21. Willett W (2013) Nutritional Epidemiology, 3rd ed. New York, NY: Oxford University Press.

22. Vance TM, Azabdaftari G., Pop EA, et al. (2015) Thioredoxin 1 in prostate tissue is associated with Gleason score, erythrocyte antioxidant enzyme activity, and dietary antioxidants. Prostate Cancer 2015, Article ID 728046.

23. Akinloye O, Adaramoye O \& Kareem O (2009) Changes in antioxidant status and lipid peroxidation in Nigerian patients with prostate carcinoma. Pol Arch Med Wewn 119, 526-532.

24. Aydin A, Arsova-Sarafinovska Z, Sayal A, et al. (2006) Oxidative stress and antioxidant status in non-metastatic prostate cancer and benign prostatic hyperplasia. Clin Biochem 39, 176-179.

25. Zhang J, Dhakal IB, Lang NP, et al. (2010) Polymorphisms in inflammatory genes, plasma antioxidants, and prostate cancer risk. Cancer Causes Control 21, 1437-1444.

26. Zhang J, Dhakal IB, Greene G, et al. (2010) Polymorphisms in hOGG1 and XRCC1 and risk of prostate cancer: effects modified by plasma antioxidants. Urology 75, 779-785.

27. Morrow JD \& Roberts LJ (1997) The isoprostanes: unique bioactive products of lipid peroxidation. Prog Lipid Res 36, 1-21.

28. Draper HH, Squires EJ, Mahmoodi H, et al. (1993) A comparative evaluation of thiobarbituric acid methods for the determination of malondialdehyde in biological materials. Free Radic Biol Med 15, 353-363.

29. Shan W, Zhong W, Zhao R, et al. (2010) Thioredoxin 1 as a subcellular biomarker of redox imbalance in human prostate cancer progression. Free Radic Biol Med 49, 2078-2087.

30. Arner ES \& Holmgren A (2006) The thioredoxin system in cancer. Semin Cancer Biol 16, 420-426.

31. Singh SS, Li Y, Ford OH, et al. (2008) Thioredoxin reductase 1 expression and castration-recurrent growth of prostate cancer. Transl Oncol 1, 153-157.

32. Holmgren A \& Lu J (2010) Thioredoxin and thioredoxin reductase: current research with special reference to human disease. Biochem Biophys Res Commun 396, 120-124.

33. Raninga PV, Trapani GD \& Tonissen KF (2014) Cross talk between two antioxidant systems, thioredoxin and DJ-1: consequences for cancer. Oncoscience 1, 95-110. 